\title{
Halle als kompakte Stadt
}

Günstige Voraussetzungen von Raum- und Infrastruktur für die Verkehrspolitik zeichnen Halle an der Saale aus. Mit vielfältigen Leitbildern wurden Ziele wie die "Nahverkehrsstadt" verfolgt. So ist es Halle bis heute gelungen, gute Erreichbarkeit mit Wirtschaftlichkeit zu vereinen.

$\mathrm{H}$

Von Werner Draeger und Dorothée Klöckner Wende durchweg gute Voraussetzungen für die Realisierung eines Konzepts ökologisch verträglicher Mobiltät. Deshalb wurde Halle im Rahmen des Forschungsprojekts „ökologisch verträgliche Mobilität“ des BMBF als Beispielstadt ausgewählt. Allerdings sind diese günstigen Voraussetzungen zunehmend gefährdet. Die Ausgangssituation von Raum- und Infrastruktur kann wie folgt beschrieben werden:

- Kompakte Siedlungsstruktur. Zwei Drittel der Bevölkerung im Raum Halle (Stadt und Umland) wohnten in Siedlungsformen (gründerzeitliche Innenstadt, sozialistische Großwohnsiedlung), die ursprünglich weitgehend autofrei konzipiert wurden.

- Die Stadt verfügt über ein dichtes Straßenbahnnetz, eine innerstädtische S-Bahnstrecke und sechs radial zum Hauptbahnhof verlaufende Eisenbahnlinien. Ehemalige Garnisonen und Industriegebiete bieten größere zusammenhängende Konversionsflächen im Stadtgebiet mit gegebener oder erweiterungsfähiger ÖV-Erschließung.

- 30 Kilometer rund um Halle gibt es mit Ausnahme von Merseburg (mit den Chemiewerken Buna und Leuna) keine größeren Industrievororte oder Kleinstädte. Wohnsuburbanisierung war noch kaum feststellbar, Gewerbesuburbanisierung fand vor allem an den Stadteingängen entlang der Bundesstraßen und der Autobahnstrecke zwischen Halle und Leipzig statt.

\section{Verkehrsplanung nach der Wende}

Nach der Wende stieg die Kfz-Nutzung und damit der Problemdruck in der gesamten Stadt stark an. Hohe Erwartungen und besondere Anforderungen wurden an die Verkehrsplanung gerichtet. Diesen versuchte die Kommune mit ersten Beschlïssen 1991 zur Orientierung der Verkehrsplanung gerecht zu werden:
- Parkraumbewirtschaftung über eine Parkraumsatzung mit Verhinderung großer Parkhäuser für Kaufhäuser in der Innenstadt; Beschleunigung für Busse und Bahnen; Festlegung eines Grundkonzeptes von $\mathrm{P}+\mathrm{R}$ für Halle; autofreie Innenstadt (10. Juli 1991)

- Beitritt der Stadt Halle in das „Gesunde-Städte-Netzwerk" der WHO (10. November 1991)

- Gesamtverkehrskonzeption als Grundlage zur Ausarbeitung des Verkehrsentwicklungsplanes der Stadt Halle (18. September 1991)

Wesentliche Teile der Gesamtverkehrskonzeption wurden aus dem Verkehrspolitischen Leitbild des „Runden Tisches Verkehr“ der Stadt übernommen. Danach sollte eine sinnvolle Aufgabenteilung zwischen allen Verkehrsarten und Verkehrsmitteln gewährleistet sein. Der ÖPNV hat bei Verkehrserschließung der Stadt und ihres Umlandes Priorität. Fußgänger- und Radverkehr werden als umweltfreundliche Verkehrsarten besonders gefördert. Die Zahl der Parkplätze innerhalb des Altstadtringes soll begrenzt gehalten werden und vor allem den Anwohnern zur Verfügung stehen. Und unter Beachtung und Erhaltung der Stadtstruktur und der Prinzipien des Biotopverbundes soll unter geringstmöglichem Landschaftsverbrauch die Konzentration des Bundesstraßennetzes durch das Zentrum von Halle reduziert werden.

\section{Planung und Umsetzung}

In einer integrierenden Arbeitsweise wurden vom Planungsring Halle ab 1992 der Flächennutzungsplan, der Landschaftsplan und der Verkehrsentwicklungsplan (VEP) parallel bearbeitet. Herausragendes Ergebnis des öffentlichen Verkehrsforums 1995 zum VEP war, daß Halle sich selbstbewußt als „Nahverkehrsstadt" versteht.

Zuvor gab es als Ergebnis von Detailarbeiten im Rahmen des VEP allerdings schon weitere richtungsweisende Beschlüsse:

Die 1992 verabschiedete Stellplatzsatzung schränkte die Stellplatzforderungen in der Altstadt auf zehn Prozent und im Innenstadtgürtel

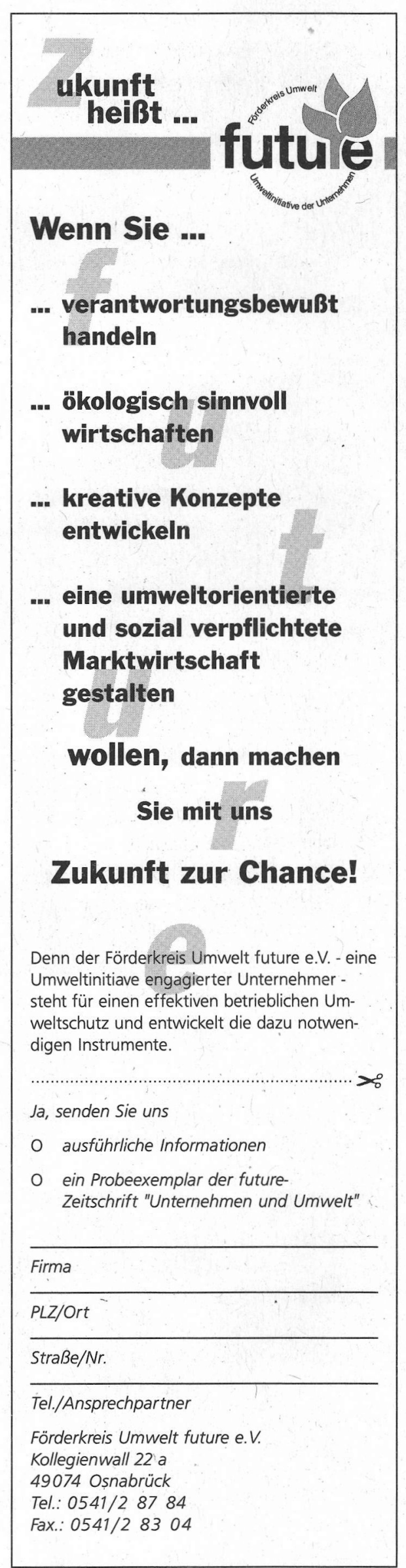


Verkehrsmittelwahl der Wohnbevölkerung von Halle. Gesamtverkehr (Ziel-Modal-Split)

\begin{tabular}{|c|c|c|c|c|c|c|}
\hline $\begin{array}{l}\text { Verkehrsmittel-Anteil in } \\
\text { Prozent Gesamtverkehr }\end{array}$ & $\begin{array}{l}1991 \\
(S+V)\end{array}$ & $\begin{array}{l}1994 \\
\text { (SrV) }\end{array}$ & $\begin{array}{l}1991 \\
\text { (KONTIV) }\end{array}$ & $\begin{array}{l}1994 \\
\text { (KONTIV) }\end{array}$ & $\begin{array}{l}\text { Ziel } 2002 \text { (Nah- } \\
\text { verkehrsplan) }\end{array}$ & $\begin{array}{l}\text { Ziel } 2010 \text { (Verkehrs- } \\
\text { politisches Leitbild) }\end{array}$ \\
\hline Bus und Bohn & 23 & 23 & 20 & $19=$ & 20 & 22 \\
\hline zu Fuी & 37 & 31 & 34 & 27 & 27 & 29 \\
\hline Fahrrad & 6 & 10 & 10 & 10 & 13 & 15 \\
\hline Pkw und motorisiertes Zweirad & 34 & 36 & 36 & 44 & 40 & 34 \\
\hline
\end{tabular}

Die ersten beiden Erhebungen 1991 und 1994 wurden nach dem System reprösentativer Verkehrsbefragungen (SVR) durchgeführt, die zweiten beiden mittels einer kontinuierlichen Verkehrserhebung in Deutschland ( KONTIV). Die Prognosen für 2002 und 2010 wurden anhand der KONTIV-Daten erstellt.

auf 30 Prozent der Richtzahlenliste der LBauO ein. Die Radverkehrskonzeption wurde im August 1995 beschlossen und in Formmaßnahmen- und umsetzungsorientierter Routenkonzepte für sieben stadtweite Routen und den Saale-Radwanderweg vertieft.

Als erste große Verkehrsbaumaßnahme wurde eine neue Straßenbahn-Ost-West-Achse zwischen Hauptbahnhof/Riebeckplatz und HalleNeustadt/Nietleben (Kostenvolumen etwa 200 Millionen Mark) verbunden mit einem Komplett-Umbau des neuralgischen Riebeckplatzes, sowie die sogenannte Dieselbrücke als erstes Teilstiick der Osttangente beschlossen. Interessant ist, daß mit dem neuen Verkehrspolitischen Leitbild von 1996 für das Modal-Split im Jahr 2010 die Wiedererlangung der Verhältnisse von 1991 angestrebt wird. Ein Kontrollverfahren soll die Wirkung von Maßnahmen auf den ModalSplit überprüfen. Konkret beziffert werden auch Minderungsziele im Rahmen des Immissionsschutzes. Hintergrund dafiur ist unter anderem der Beschluß der Stadt, dem „Klima-Bündnis der Europäischen Städte" beizutreten (Dezember 1992). Die Verkehrskonzeption Altstadt wurde vorab bereits beschlossen (9/96). Das Ziel der ,autoarmen“ Altstadt ist damit greifbar nahe und im Südteil bereits Realität.

\section{Anspruch und Widersprïche}

Obwohl dem ÖPNV als System oberste Priorität eingeräumt wird, sind die Bauvorhaben und Begleitmaßnahmen für unterschiedliche Verkehrsarten untereinander nicht abgewogen. Zwangsläufig divergieren die Interessen zwischen professionellen Akteuren, die die Problemlösung nur im Bau neuer Straßen und Tiefgaragen sehen, und Akteuren, die die Chancen der kompakten Stadt mit kurzen Wegen durch die Förderung der umweltfreundlichen Verkehrsarten nutzen wollen. Das für einen umfassenden Konsens gedachte Leitbild droht zwi- schen diesen Interessenkonflikten zerrissen zu werden. Selbst die Konsens-These „Halle ist Nahverkehrsstadt" wird nun mit dem Argument unterhöhlt, der Nutzen gebauter Straßen sei höher als der Nutzen gebauter Schienenstrecken.

Weitere Anzeichen für Diskrepanzen zwischen Leitbild und Umsetzung in der Planung sind bereits bei laufenden Vorhaben auszumachen:

- Zur Beschleunigung des Straßenbahnverkehrs im Zuge der Magdeburger Chaussee und der Trothaer Straße (B6) sollte die Straßenbahn einen eigenen Gleiskörper erhalten. Der konkrete Baubeschluß wurde aber abgelehnt, um den motorisierten Individualverkehr vierstreifig führen zu können.

- Entwurf und Betrieb von Straßenknoten richten sich im Planeralltag nach den Maximen Beschleunigung von Straßenbahn und Bus sowie größtmögliche Leistungsreserven für den Kfz-Verkehr. Die Ansprüche des Fuß- und Fahrradverkehrs finden sich entgegen der Ziele des alten und des neuen verkehrspolitischen Leitbildes nur marginal wieder.

- Die Verkehrs-Zuständigkeit der drei Dezernate (Inneres, Bauen sowie Planen und Umwelt) führt zu langwierigen Entscheidungsfindungsprozessen (Ämterabstimmung). Die Trennung in Ausfiuhrung und Anordnung sorgt für hohe Reibungsverluste nach innen und außen und hemmt die Umsetzung

\section{Veränderungen in der Stadt}

Der Beitritt Halles zum Klimabündnis und zum Gesunde-Städte-Netzwerk hat bei den Gruppen Hoffnung geweckt, die sich seit der Wende für einen ökologisch verträglichen Verkehr einsetzen. Dieser Anspruch wird wegen der fehlenden Umsetzung aber faktisch nicht erfïllt.

Die Wohnsuburbanisierung im Saalkreis hat sich verstärkt. Der Motorisierungsgrad erhöhte sich, allerdings mit Konsolidierungstendenz. Der Bau von Einkaufszentren im Außenbereich und in Umlandgemeinden setzte sich fort, während Geschäfte in innerstädtischen Quartieren schließen, eine wohnstandortnahe Versorgung gerade für ältere Bewohner ist damit gefährdet. Nach Klärung von Restitutionsansprüchen stehen nun auch in der Innenstadt Flächenreserven für den Handel zur Verfiigung. Potentielle Investoren versuchen aber weiterhin, der Stadt ihre Kfz-orientierten Vorstellungen (massiven Stellplatzbau mit Aushebeln der Stellplatzsatzung) aufzudrücken.

Auch die mehrheitlich in Außenbezirken liegenden Gewerbestandorte und -parks wurden $\mathrm{zu}$ großziigig geplant und verwirklicht. Stagnation in der Innenstadt und ungeordnete Entwicklung an Stadtrand und im Umland wurden trotz gegenteiliger Absichten durch den in Halle hohen Anteil ungeklärter Eigentumsverhältnisse gefördert.

\section{Aktuelle Chancen für die kompakte Stadt Halle}

Die zu Projektbeginn formulierten Ziele lassen sich gewiß nicht wie geplant erreichen. Trotz der günstigen Raumstruktur führten die rasante Motorisierung und die Übernahme ,westlicher“ Lebensweisen zu hohen Belastungen, so daß die autoreduzierte Stadtnutzung in der Modellstadt in Frage gestellt ist und insofern nicht exemplarisch auf andere Städte übertragen werden kann. Auch in Halle führt der Weg offensichtlich über erlebte Erfahrung.

Eine Chance besteht im Sinne der Projektintentionen in den knappen Finanzmitteln. Großinvestitionen sollen auf die ÖPNV-Förderung (wie zum Beispiel die neue Straßenbahnlinie nach Halle-Neustadt) konzentriert werden. Da Fußund Radverkehr vergleichsweise sehr niedrige Investitions- und Betriebskosten verursachen, könnten diese Verkehrsarten mit relativ geringem finanziellen Aufwand gefördert werden.

Vom politischen Willen und der konsequenten Umsetzung planerischer Maßnahmen hängt es ab, ob die beschlossene ÖPNV-Bevorrechtigung in Halle Realität wird oder ob eher die Erhaltung und Erweiterung der Kfz-Leistungsfähigkeit zu Lasten des nichtmotorisierten Verkehrs erfolgt. Autoreduzierte Stadtnutzung steht und fällt mit diesen Fortbewegungsarten.

\section{Die Autorlnnen}

Dr.-Ing. Werner Draeger; Dorohhée Klöckner, Stud.-Ass'; tätig im Büro für integrierie Stadłund Verkehrsplanung (BiS) Bonn, Colmantstr. 5 , 53115 Bonn, Tel. $(0228) 697401$ 
(c) 20I0 Authors; licensee IÖW and oekom verlag. This is an article distributed under the terms of the Creative Commons Attribution Non-Commercial No Derivates License (http://creativecommons.org/licenses/by-nc-nd/3.o/), which permits unrestricted use, distribution, and reproduction in any medium, provided the original work is properly cited. 